\title{
Alternativa de financiación basada en titularización para la producción de plátano en el Urabá antioqueño
}

\section{Securitization-based financing method for plantain production in the Urabá region of Antioquia}

\author{
Juan Camilo Lopera-Lopera a, Luisa Fernanda Avalos-Hincapié b, \& Eduardo Alexander \\ Duque-Grisales ${ }^{c}$ \\ a Institución Universitaria ESUMER, Medellín, Colombia, juan.lopera@esumer.edu.co \\ b Institución Universitaria ESUMER, Medellín, Colombia. luisa.avalos@esumer.edu.co \\ c Institución Universitaria ESUMER, Grupo GIDE, Medellín, Colombia, eduardo.duque@esumer.edu.co
}

Recibido: septiembre 18, 2019. Aceptado: noviembre 19, 2019.

\begin{abstract}
Resumen
Los procesos de globalización a los que se enfrenta el sector agropecuario en Colombia conllevan una etapa de reestructuración para ser más competitivos y llegar a nuevos mercados, dado que los consumidores hoy en día son cada vez más informados y exigentes en cuanto a la calidad esperada de los productos. Esto lleva a que las empresas sean más eficientes e innovadores en los procesos de producción y comercialización, así como en la diversificación de sus portafolios de productos y mercados. Sin lugar a duda, éste es uno de los mayores desafíos enfrentados por el sector agro en Colombia y para ello se requiere un incremento de las tasas actuales de inversión, favoreciendo el impulso tecnológico y ofreciendo atención prioritaria a la búsqueda y adopción de nuevas alternativas de financiación. En este artículo se resalta el potencial de Colombia en la producción de plátano y se hace énfasis en la necesidad de contar con mecanismos innovadores de financiación para afrontar los problemas actuales de escasez de recursos económicos. Para ello se propone la financiación de productores de plátano a través de la Titularización, con el fin de fortalecer la competitividad del sector y poder aumentar su participación en las exportaciones colombianas.
\end{abstract}

Palabras Clave: Titularización, Mercado de capitales, Sector agropecuario, Productores de Plátano, Mecanismos de financiación.

\begin{abstract}
The globalization processes faced by the agricultural sector in Colombia entail a restructuring process to be more competitive and reach new markets, given that consumers today are increasingly informed and demanding in terms of expected product quality. These requirements lead to companies being more efficient and innovative in the production and marketing processes, as well as in the diversification of their product and market portfolios. Undoubtedly, this is one of the main challenges faced by the agricultural sector in Colombia, leading to an increase in the current levels of investment, promoting technological improvements, and giving higher priority to the implementation of new financing mechanisms. This article highlights the potential of Colombia in plantain production and emphasizes the need for innovative financing mechanisms to address the current problems of economic resource shortages. In this work, Securitization arises as an alternative financing method for plantain producers, looking to strengthen the competitiveness of the sector and increase its participation in Colombian exports.
\end{abstract}

Keywords: Securitization, Capital Market, Agricultural Sector, Plantain Producers, Financing Mechanisms.

\section{INTRODUCCIÓN}

El agro en Colombia es un sector que ha venido creciendo a lo largo de los años y se puede evidenciar ya que, con cifras presentadas por el Ministerio de Agricultura durante el proceso de empalme presidencial [1], "entre el año 2010 y el año 2017, el crecimiento del valor del PIB agropecuario fue de $25 \%$, al pasar de $\$ 47,1$ billones a $\$ 58,9$ billones" lo que evidencia que es un sector que viene tomando fuerza y que cada vez más aporta al PIB del país. Adicionalmente, en los últimos 8 años este sector ha generado 290.000 puestos de trabajo y ha sacado de la pobreza a 1.600 .000 de personas [1]. Sin embargo, este es un sector que podría crecer mucho más, dado que en Colombia se cuenta con una geografía que permite tener gran diversidad en los productos que puedan extraerse de la tierra. Uno de estos productos es el plátano el cual es el tercer producto más exportado en Colombia $(3.1 \%)$, luego del petróleo $(47.9 \%)$ y el café $(7.8 \%)$ y es el segundo producto agrícola que más se produce en el país, específicamente en mayor cantidad en el Urabá Antioqueño, dadas las características climáticas y geográficas de esta área.

\section{Citar como:}

J. Lopera, L. Ávalos, E. Duque. “Alternativa de financiación basada en titularización para la producción de plátano en el Urabá antioqueño” Revista CINTEX, Vol. 24(1), pp. 41-50. 2019. 
El sector de cultivadores del plátano en Colombia enfrenta retos como las dificultades en aspectos fitosanitarios, y la escasa inversión en prácticas agrícolas como riego, renovación, drenaje y fertilización; todos los anteriores son elementos que impactan la competitividad, calidad y productividad del cultivo con respecto a mercados internacionales [2]. Esta situación obliga a la búsqueda de un mejoramiento general de aquellos factores de producción manipulables dentro del proceso de cultivo de plátano, a través del desarrollo e implementación de prácticas agronómicas innovadoras [3].

En este mismo sentido, las empresas que se dedican a la producción de plátano no han logrado explotar todo su potencial y sus capacidades competitivas, dadas las dificultades que encuentran en el momento de capitalizarse, ya que en el mercado no existen mecanismos de financiación que apoyen e impulsen el crecimiento de este sector, ofertando tasas que no son acordes a la realidad del agro y lo único que logran es incrementar el porcentaje de gasto financiero en el que incurren las empresas [4]. El gobierno nacional ha tratado de implementar algunas medidas para apoyar a pequeños productores de plátano, pero no han sido eficaces o suficientes para que los agricultores no tengan la necesidad de ir a buscar créditos con tasas de interés muy altas, que afectan sus ingresos y disminuyen su competitividad frente al mercado nacional e internacional.

Por ende, el financiamiento de las cosechas se ha convertido en los últimos años en la piedra angular de las industrias plataneras no solo en el Urabá Antioqueño, sino también a nivel nacional, siendo el principal aspecto que determina que la inversión en este tipo de producto agrícola sea viable frente a los grandes riesgos que este representa. Se ha logrado evidencia que una errónea gestión limita las probabilidades de obtener buenos ingresos al momento de realizar la recogida de las cosechas. Además de lo anterior, se logra evidenciar que la falta de conocimiento sobre los mecanismos de financiación con los que se cuenta en el país genera que en su mayoría las medianas y pequeñas empresas dedicadas a la producción de plátano en el Urabá Antioqueño, incurran en grandes porcentajes de deuda, incrementando su gasto financiero y por ende comprometiendo uno de los pilares fundamentales de las finanzas el cual indica que los negocios o compañías sean duraderas en el tiempo.

Es por todo esto que este artículo hace referencia a la Titularización como solución a los problemas de financiación y de liquidez que presentan las empresas que producen plátano en Colombia, brindando una nueva alternativa diferente a la banca tradicional que es la única posibilidad que encuentran en el mercado financiero las compañías agrícolas del país [5]. La titularización es un proceso de emisión de valores basado en el conjunto de activos similares que sirven como garantía. Las razones para aplicar la titularización de activos son numerosas y la más importante es la diversificación de las fuentes de financiación, la cual posee numerosas ventajas para los inversionistas y quienes ofrecen el título [6]. La experiencia de los países desarrollados muestra que la titularización de activos puede ser una fuente importante de financiamiento para la agricultura [7]. Dentro de la metodología considerada en este trabajo de investigación sobre los agricultores del plátano en la región de Urabá en Antioquia, se deben tener en cuenta aspectos que son requerimientos del cultivo tales como el clima y el terreno, y aspectos sociales como la política y el nivel de seguridad, entre otros, ya que todos estos factores son importantes para determinar el nivel y tipo de riesgos asociados a los activos para la titularización.

$\mathrm{Al}$ analizar cómo se puede introducir un marco de financiación orientado al mercado respaldado por un mecanismo de titularización para los agricultores de cultivos de plátano, este documento contribuye a la literatura existente de dos maneras. Por un lado, representa el primero de su tipo en el área de financiamiento de cultivos de plátano en Colombia. Hasta donde sabemos, no se han publicado trabajos en lo que respecta a la aplicación de la titularización al caso específico de la financiación de plátano en el Urabá antioqueño. Por otro lado, proporciona una plataforma conceptual preliminar que se utilizará para futuras investigaciones en este campo. De lo anterior, se desprende la importancia de este estudio para que los agricultores puedan encontrar nuevas formas de apalancamiento financiero. Conociendo las consecuencias financieras de la titularización, los cultivadores de plátano podrán determinar las ventajas y desventajas de este mecanismo con respecto a las alternativas de financiación tradicional, ampliando la base de herramientas para solucionar las dificultades de financiación, competitividad y productividad [8].

El artículo está organizado como se explica a continuación. La sección dos presenta una exploración de la literatura acerca de la producción de plátano en Colombia. El capítulo 3 describe la metodología empleada en el estudio para la definición del procedimiento específico para el uso de la Titularización en las empresas del sector agropecuario. Para finalizar, se presentan tanto el análisis de resultados como las conclusiones. 


\section{CONTEXTO DE LA PRODUCCIÓN DE PLÁTANO EN EL URABÁ ANTIOQUEÑO}

\subsection{Cadena agroalimentaria del plátano en Colombia}

Como todas las actividades económicas, la producción agropecuaria está conformada por diferentes factores, todos esenciales y con funcionalidades particulares dentro de la cadena de producción. Según Hurtado [9],

"La cadena Agroalimentaria del plátano en Colombia está conformada por los productores, comercializadores, industrias de procesamiento, productores de semilla, proveedores de insumos, exportadores, universidades, centros de investigación e instituciones regionales y Nacionales que acompañan el proceso como el Instituto Colombiano Agropecuario - ICA, Servicio Nacional de Aprendizaje - SENA, Universidad Nacional Abierta y a Distancia - UNAD, Centro Internacional de Agricultura Tropical - CIAT, Asociación Hortofrutícola de Colombia - Asohofrucol, secretarías de agricultura y corporaciones autónomas regionales, entre otras. La cadena del plátano se caracteriza por la intervención institucional que ha sido determinante en su disposición y desarrollo, se cuenta con asistencia técnica con el fin de colaborar con la organización de los productores, financiamiento y canales de comercialización. En la cadena del plátano se cuenta con 4 eslabones básicos: Agricultores (Productores primarios), comercializadores, industriales y exportadores. Adicionalmente, existen actividades económicas ligadas que respaldan los servicios de apoyo necesarios para el buen desarrollo de la cadena del plátano. El producto fresco se obtiene en las fincas y luego de ser cosechado, inspeccionado, seleccionado y clasificado, se lleva al mercado interno y al mercado internacional, ya sea para su consumo o para ser usado como materia prima en la fabricación de plátanos congelados, pasabocas o alimentos para animales." [9]

\subsection{Caracterización de los productores de plátano}

Para hablar de la situación de la plantación de los cultivos de plátano en el Urabá Antioqueño, es necesario hablar un poco del por qué se eligió esta área y de su ubicación geográfica (ver Figura 1) como se presenta a continuación:

"El Urabá Antioqueño es la región costera del departamento de Antioquia, la cual es zona fronteriza de Panamá, océano Pacífico y Atlántico, y con la región urabaense chocoana y cordobés. Se encuentra compuesta de 11 municipios divididos en tres zonas:

Zona norte: Arboletes, San Juan de Urabá, San Pedro de Urabá y Necoclí

Zona centro: Apartadó, Carepa, Chigorodó y Turbo

Zona sur: Mutatá, Murindó y Vigía del Fuerte

La zona de Urabá Antioqueño cuenta con una población de 693.868 habitantes, donde el 51,4\% se encuentra registradas como víctimas del conflicto armado, pues allí se implementó el segundo laboratorio paramilitar del país y según el Centro Nacional de Memoria Histórica, hubo10 mil asesinatos entre los años 1985 y 2011." [10]
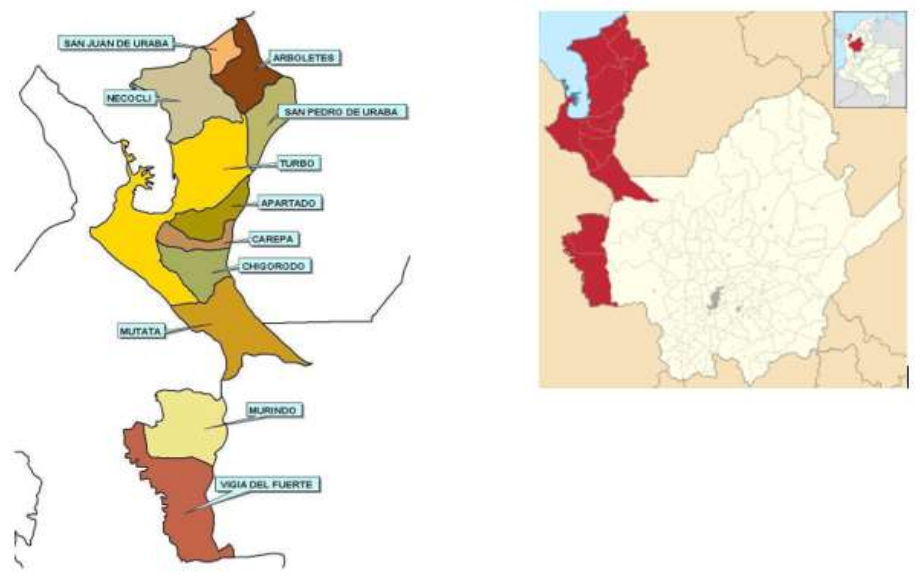

Figura 1. Ubicación geográfica del Urabá Antioqueño. 
Urabá constituye la zona platanera y bananera más importante de Colombia; este producto mantiene el tercer lugar en la agroindustria de la región, con unas 65.000 hectáreas de sembrados distribuidos entre plátano y banano; adicionalmente, la calidad de los suelos en la región del Urabá permite que también se cultive piña, cacao, yuca, palma y ají, entre otros productos [11]. En la tabla 1 se presentan las ventajas y desventajas que presenta el Urabá antioqueño, con respecto a la producción de plátano.

TABla 1. Ventajas y desventajas del URABá AntiOQUeÑo

\begin{tabular}{|c|c|}
\hline Ventajas & Desventajas \\
\hline $\begin{array}{l}\text { - } \text { Calidad de los suelos, al poder producir } \\
\text { gran cantidad de productos agrícolas. } \\
\text { Internacionalización, el plátano es el } \\
\text { tercer producto que más se exporta en } \\
\text { el país. } \\
\text { Producción a gran escala, la zona } \\
\text { cuenta con grandes proporciones para } \\
\text { implementar una producción a grandes } \\
\text { rasgos. } \\
\text { Mano de obra, en esta zona del } \\
\text { departamento la tasa de desempleo es } \\
\text { más alta que en el resto, por lo que se } \\
\text { podrían generar más empleos. } \\
\text { Rentabilidad del agro, esta es una de } \\
\text { las actividades más rentables en el } \\
\text { mercado a pesar de sus costos de } \\
\text { producción. } \\
\text { Zona fronteriza, con Panamá, océano } \\
\text { Pacífico y Atlántico, y con la región } \\
\text { urabaense chocoana y cordobés. }\end{array}$ & $\begin{array}{l}\text { - Inseguridad, los altos índices de } \\
\text { violencia en la zona. } \\
\text { Riesgo, las tasas de interés se ven } \\
\text { afectadas por los temas de seguridad. } \\
\text { - Poco desarrollo urbano, no es tan } \\
\text { factible encontrar en el sector un gran } \\
\text { desarrollo. } \\
\text { - Mano de obra no calificada, el } \\
\text { desarrollo académico de esta parte del } \\
\text { departamento no es muy avanzado. } \\
\text { - Desplazamiento de los jóvenes } \\
\text { campesinos a las grandes ciudades. }\end{array}$ \\
\hline
\end{tabular}

\section{MECANISMOS ALTERNATIVOS DE FINANCIACIÓN: TITULARIZACIÓN EN COLOMBIA}

La titularización es un esquema de financiamiento consistente en la transformación de activos o bienes en valores negociables por medio de un contrato de fiducia, en los mercados de capitales para obtener liquidez [7], [12], [13]. En general, todos los activos que generan flujo de efectivo futuro son sujetos de titularización.

Según Blanco Barón, "la titularización incluye los procedimientos para emitir papeles en oferta pública a partir de subyacentes específicos, lo que requiere un conocimiento especializado de la estructuración de emisiones con este fin, y una organización en la que interactúan los agentes que realizan ciertas actividades en el mercado de valores para producir documentos o instrumentos financieros negociables" [14].

En Colombia se han tenido experiencias exitosas en la aplicación de la Titularización como mecanismo de financiación [15]. Una de ellas ha sido la titularización de ganado de engorde, por un valor aproximado de 38 millones de dólares para el año 2003 [16]. En este caso de titularización, un conjunto de ganaderos interesados en engordar su ganado conformó un patrimonio autónomo con alrededor de 152.000 reses, con el fin de emitir títulos de contenido crediticio; la experiencia fue positiva para todas las partes, generando el capital para el pago a los inversionistas y el cubrimiento de los costos, y un remanente como utilidad de la ceba de los animales.

\subsection{Proceso de Titularización del plátano}

En la estructuración de titularizaciones participan los siguientes agentes [17] [18]:

- El originador o dueño de los activos o derechos titularizables (subyacentes): Persona natural o jurídica que transfiere los bienes y activos o flujos de caja presentes y futuros de una actividad dentro de un Patrimonio Autónomo, mediante la firma de un contrato de fiducia mercantil irrevocable. Es el encargado de entregar el activo subyacente (cultivo de plátano) el cual será titulado.

- Estructurador: Entidad encargada del diseño técnico, jurídico, financiero y operativo del proceso de titularización, por encargo directo del originador. 
- Agente de Manejo: Sociedad fiduciaria que emite los nuevos valores en representación del Patrimonio Autónomo, en su condición de administrador del fideicomiso, que recauda los recursos provenientes de dicha emisión y que se relaciona jurídicamente con los inversionistas. En Colombia, actúan como agentes de manejo las sociedades fiduciarias y las instituciones financieras. Las sociedades comisionistas de bolsa pueden también ser agentes de manejo, por medio de fondos de valores.

- Administrador de la Emisión: Entidad encargada de la conservación, custodia y administración de los títulos valores producto del proceso de titularización. Debe llevar el libro de registro de los inversionistas y actuar como agente de pago de las obligaciones del patrimonio. En el caso colombiano, esta función la lleva a cabo el Depósito Centralizado de Valores - Deceval.

- Colocador: Entidad que se encarga de colocar los títulos en el mercado. Pueden ser colocadores todos los intermediarios inscritos en el registro nacional de valores.

- Sociedad calificadora de riesgos: Realizan el estudio de riesgos del proceso, con el fin de otorgar una calificación que sirva como referencia para los inversionistas acerca de las posibilidades de pago de los títulos adquiridos.

Una titularización exitosa requiere del cumplimiento de unos prerrequisitos básicos, tales como que el instrumento sea atractivo tanto para los inversionistas (quienes proveen el capital) como para el originador del proyecto [19]. Para esto, es importante que los rendimientos de los títulos emitidos puedan contar con una parte variable y una fija. Por lo que los títulos deberán estar indexados a la DTF + Spread. Sin embargo, para el cálculo del spread que se debe ofrecer a los inversionistas se requiere de la gestión del estructurador, el cual, a través de una rueda de negocio entre los posibles inversionistas, capture cual es el apetito de riesgo hacia este tipo de instrumentos. La figura 2 presenta el modelo de titularización donde se interrelación todos los actores que intervienen en el proceso.

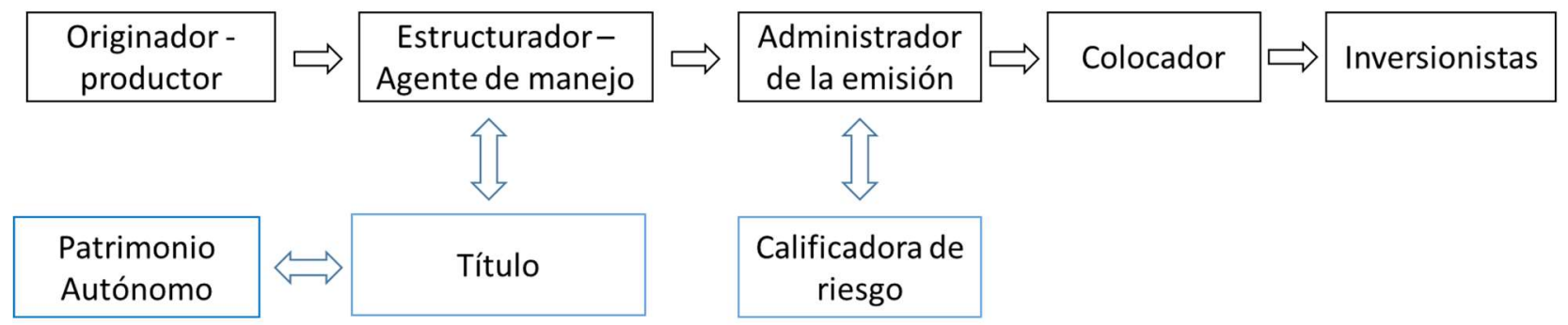

Figura 2. Modelo de titularización para productores de plátano. Fuente: Elaboración propia con base a [7], [19].

El proceso inicia cuando el originador elige un agente estructurador (Titularizadora) y traspasa sus activos para conformar el patrimonio autónomo. El agente de manejo emite los títulos que son traspasados al administrador de la emisión, quienes realizan la evaluación financiera para el inversionista y una evaluación de mercado (precolocación). En esta etapa se realiza el envío de documentación a la superintendencia de valores y a la agencia calificadora de riesgos. Después de que se presenta la documentación requerida por las entidades calificadoras y supervisoras, estas emiten un juicio realizando observaciones sobre el procedimiento, con el fin de que el estructurador ejecute las modificaciones correspondientes y comience a negocias con el colocador una vez se apruebe la oferta pública, para proceder a la emisión y colocación con los inversionistas.

\subsection{Caso de estudio}

Dentro de la ejecución de este trabajo investigativo exploratorio, se realizó un estudio de caso de aplicación para determinar la información económica y financiera de las empresas productoras de banano en el Urabá antioqueño y las fuentes de financiación empleadas por estas, para que se muestren las consecuencias económicas de la titularización. El bien que se quiere titularizar son los flujos futuros generados por la siembra y cosecha de los frutos del plátano para una de las empresas, más específicamente los derechos económicos sobre la cosecha de uno de estos.

Inicialmente el precio, ni la cantidad final está definida, solo se estipula la tasa de colocación que percibirán los inversionistas. El título por evaluar presenta las siguientes características:

- Monto por emitir: Variable

- Plazo de la emisión: 10 años

- Circulación: a la orden 
- Valor nominal del título: $\$ 1.000 .000$

- Tasa de colocación: IPC + puntos

- Periodicidad: TMV (Trimestre Mes Vencido)

- Precio Kilo: $\$ 1.200^{1}$

- Crédito solicitado: 1.105 millones

Según el ICA (Instituto Colombiano Agropecuario) si se implementan las nuevas tecnologías en Colombia, la producción puede alcanzar beneficios de 50.1 ton/ha (toneladas por hectárea), en este caso se buscará producir a escala para que los beneficios sean mayores. Por lo anterior y basados en los costos de este, se estima cosechar las siguientes cantidades por los 10 años donde será efectivo el título valor, aunque se estima que para este periodo se pueda tomar un total de 10 cosechas, estimando que cada una de estas se tomará un tiempo promedio de 12 meses, como expone el ICA.

Tabla 2. Unidades producidas cultivo de plátano

\begin{tabular}{|l|r|}
\hline Cantidad producida por año & Cifra \\
\hline Hectáreas & 5 \\
Toneladas & 50 \\
Kilos & 50,100 \\
To/ha & 251 \\
Kilos/ha & 250,500 \\
Precio plátano x kilo & 1,200 \\
\hline Total ingreso & $300,600,000$ \\
\hline
\end{tabular}

Para realizar la simulación es necesario tomar como referencia el banco que mejor tasa ofrece para los productores agrícolas, como lo es el Banco Agrario, este se eligió dado que es un banco dedicado solamente a facilitar financiación a las compañías agrícolas del país, mediante subsidios que son aportados por el gobierno para disminuir las tasas de interés; para el año 2019 la tasa se encuentra ubicada en 12,51\% EA.

Con el fin de determinar la efectividad de los mecanismos de financiación, se procede a realizar un comparativo entre la financiación para los cultivadores de plátano mediante tres mecanismos: crédito a cuota fija, crédito con amortización constante o por medio de la Titularización. Se realiza una proyección de los flujos de caja del proyecto y se espera titularizar los flujos de caja futuros. De igual manera, dichos flujos de caja serán el soporte para pagar los créditos del sistema tradicional (cuota fija, amortización constante). En las tablas 3 a 5 se presentan los flujos de caja del proyecto para cada uno de los mecanismos de financiación. Para la elaboración del flujo de caja del proyecto, se proyectan de forma mensual los ingresos del contrato de producción de plátano, tomando como referencia datos históricos y se descuenta a la tasa de descuento establecida por el sector platanero.

Tabla 3. Flujos de caja financiación vía Titularización (cifras en millones de pesos)

\begin{tabular}{|c|c|c|c|c|c|c|c|c|c|c|c|}
\hline Flujo de caja & 2019 & 2020 & 2021 & 2022 & 2023 & 2024 & 2025 & 2026 & 2027 & 2028 & 2029 \\
\hline Free Cash Flow & -1.381 & 136 & 147 & 158 & 168 & 180 & 191 & 204 & 218 & 232 & 239 \\
\hline + Deuda & 1.105 & & & & & & & & & & \\
\hline - Intereses & & 124 & 111 & 99 & 87 & 74 & 62 & 50 & 37 & 25 & 12 \\
\hline $\begin{array}{l}\text { + Escudo Fiscal } \\
\text { - Caja disponible }\end{array}$ & & 0 & 0 & 0 & 0 & 0 & 0 & 0 & 0 & 0 & 0 \\
\hline Amort & & 110 & 110 & 110 & 110 & 110 & 110 & 110 & 110 & 110 & 110 \\
\hline$=$ Saldo Final & & 0 & 0 & 0 & 0 & 0 & 0 & 0 & 0 & 0 & 0 \\
\hline Equity Cash Flow & -276 & -99 & -75 & -51 & -29 & 5 & 18 & 44 & 70 & 97 & 116 \\
\hline
\end{tabular}

\footnotetext{
${ }^{1}$ El precio por plátano se obtuvo de la página de Corabastos para el día 23 de octubre del 2019, se tomó como base dado que se espera que este valor incremente en los próximos años 
Tabla 4. Flujos de caja financiación vía Amortización Constante (cifras en millones de pesos)

\begin{tabular}{|c|c|c|c|c|c|c|c|c|c|c|c|}
\hline Free Cash Flow & 2019 & 2020 & 2021 & 2022 & 2023 & 2024 & 2025 & 2026 & 2027 & 2028 & 2029 \\
\hline Free Cash Flow & $\overline{1,381}$ & 136 & 147 & 158 & 168 & 180 & 191 & 204 & 218 & 232 & 239 \\
\hline + Deuda & 1,105 & & & & & & & & & & \\
\hline - Intereses & & 163 & 152 & 140 & 121 & 105 & 83 & 68 & 51 & 34 & 17 \\
\hline $\begin{array}{l}\text { + Escudo Fiscal } \\
\text { - Amortización }\end{array}$ & & $\begin{array}{l}0 \\
110\end{array}$ & $\begin{array}{l}0 \\
110\end{array}$ & $\begin{array}{l}0 \\
110\end{array}$ & $\begin{array}{l}0 \\
110\end{array}$ & $\begin{array}{l}0 \\
110\end{array}$ & $\begin{array}{l}0 \\
110\end{array}$ & $\begin{array}{l}0 \\
110\end{array}$ & $\begin{array}{l}0 \\
110\end{array}$ & $\begin{array}{l}0 \\
110\end{array}$ & $\begin{array}{l}0 \\
110\end{array}$ \\
\hline Equity Cash Flow & -276 & -138 & -116 & -92 & -64 & -36 & -3 & 26 & 56 & 88 & 112 \\
\hline
\end{tabular}

Tabla 5. Flujos de caja financiación vía Cuota Constante (cifras en millones de pesos)

\begin{tabular}{|c|c|c|c|c|c|c|c|c|c|c|c|}
\hline Flujo de caja & 2019 & 2020 & 2021 & 2022 & 2023 & 2024 & 2025 & 2026 & 2027 & 2028 & 2029 \\
\hline Free Cash Flow & -1.381 & 136 & 147 & 158 & 168 & 180 & 191 & 204 & 218 & 232 & 239 \\
\hline + Deuda & 1.105 & & & & & & & & & & \\
\hline - Intereses & & 138 & 131 & 122 & 112 & 101 & 89 & 75 & 59 & 42 & 22 \\
\hline + Escudo Fiscal & & 0 & 0 & 0 & 0 & 0 & 0 & 0 & 0 & 0 & 0 \\
\hline - Amortización & & 61 & 69 & 78 & 87 & 98 & 111 & 125 & 140 & 158 & 177 \\
\hline Equity Cash Flow & -276 & -64 & -53 & -41 & -32 & -20 & -9 & 4 & 18 & 32 & 39 \\
\hline
\end{tabular}

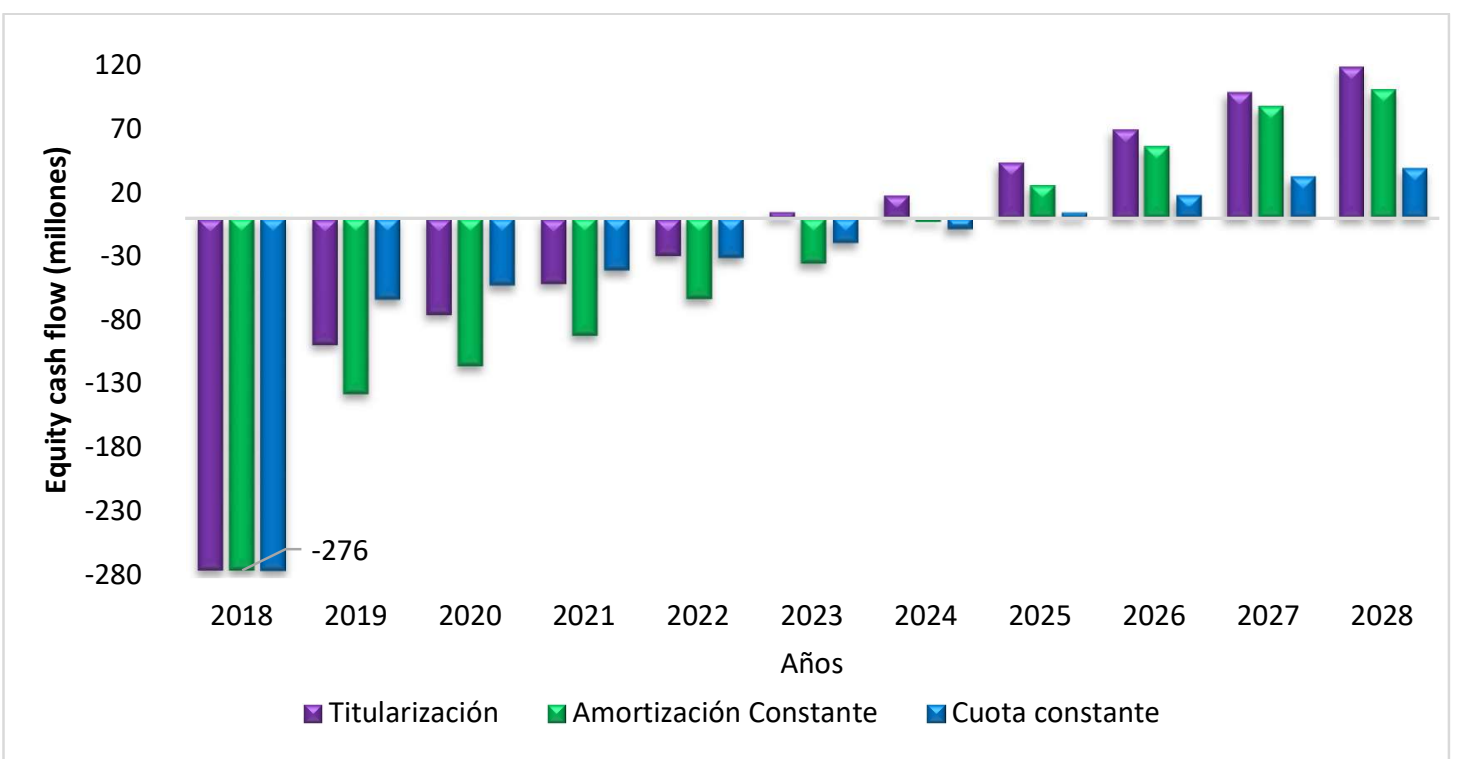

Figura 3. Flujos de caja de los modelos de financiación para productores de plátano.

En la figura 3 se presenta el comparativo gráfico de los tres sistemas de financiación para la producción de plátano. En la Figura 4 se presenta un análisis de sensibilidad de la Tasa Interna de Retorno - TIR del inversionista con respecto a la variación en la tasa de interés (spread). A medida que incrementa la tasa de interés va disminuyendo la TIR del inversionista, además de que muestra que a menores tasas la más recomendada es la Titularización, dado que alcanza mayor porcentaje de retorno al inversionista y también que es competente con los otros dos mecanismos de financiación como lo son deuda con amortización constante y deuda con cuota constante. 


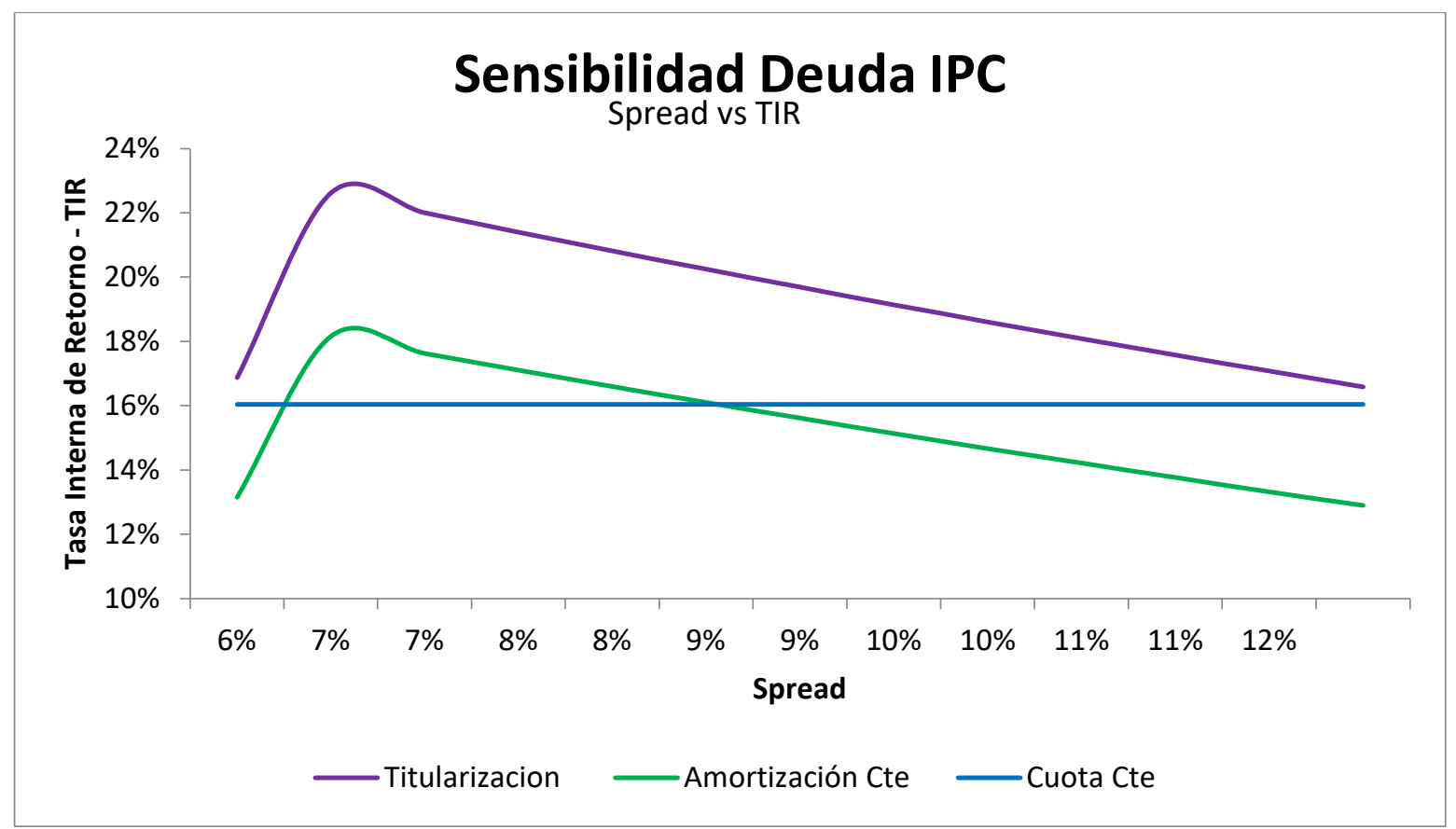

Figura 4. Simulación TIR Inversionista.

Otro aspecto importante para tener en cuenta, a la hora de elegir un mecanismo de financiación es el porcentaje de endeudamiento. En la Figura 5, se presenta la sensibilidad de los sistemas de financiación con respeto al porcentaje de deuda requerido para llevar a cabo el proyecto.

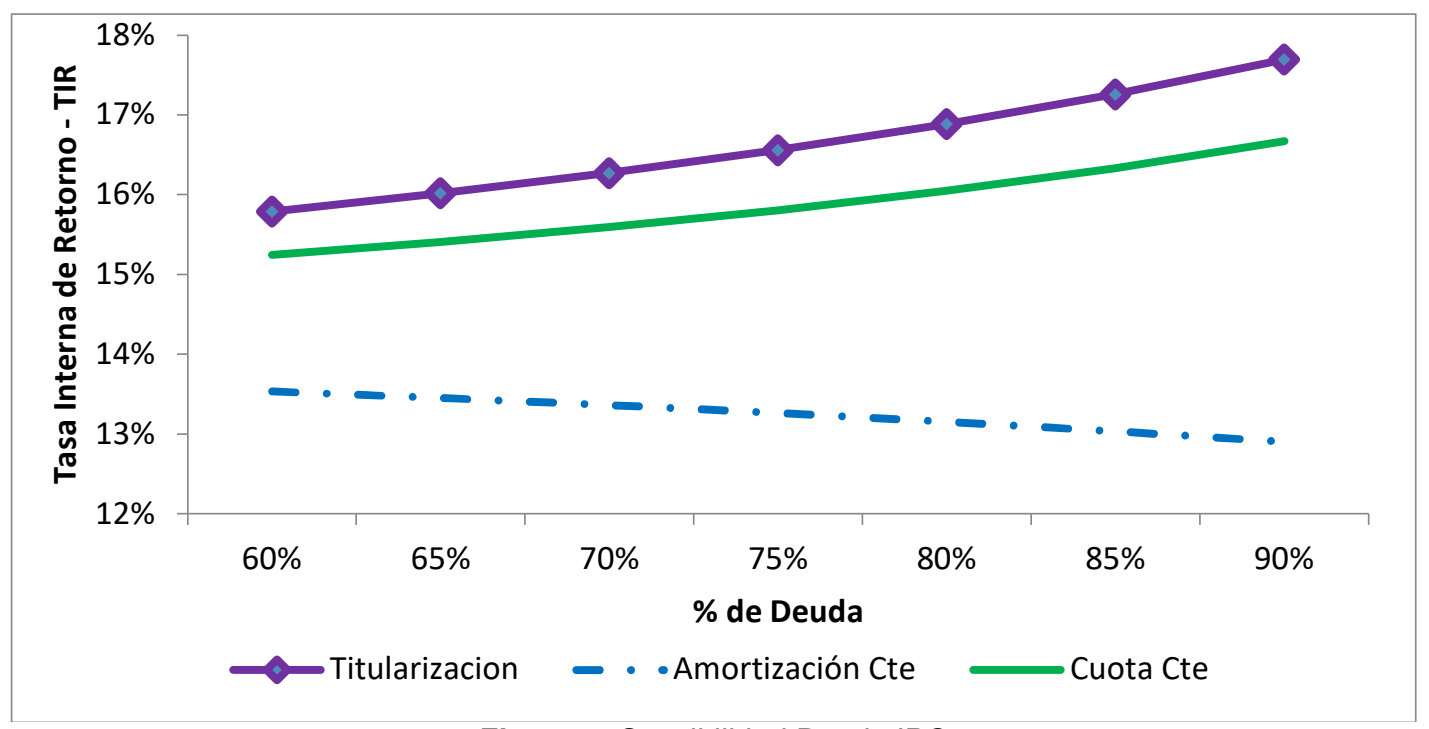

Figura 4. Sensibilidad Deuda IPC

Se puede observar que la titularización, es el mecanismo más sensible con el endeudamiento y, también encontramos que la TIR ofrecida se acerca al margen neto operacional que según el ICA se encuentra alrededor de un $17 \%$, lo que garantiza que la información con la que se ha venido trabajando es elocuente y se aproxima a la realidad del sector en nuestro país.

Con la información anterior tenemos las bases suficientes para determinar la viabilidad de la Titularización como mecanismo de financiación para la producción del plátano en el Urabá Antioqueño; con el comparativo entre financiarse con Titularización, Amortización Constante o Cuota Fija, encontramos que la titularización es el mecanismo que mayor TIR puede obtener el empresario, aunque eso depende de la tasa de interés que se encuentre en el mercado. También se debe mitigar los riesgos del inversionista, mediante un análisis de los aspectos tanto externos o internos que pongan en riesgo el éxito de la producción de plátano. Aunque también dependiendo del nivel del riesgo que esté dispuesto a asumir el agricultor, podría decantarse en financiarse por 
medio de Cuota fija, dado que por este mecanismo estaría obteniendo una TIR viable respecto a la realidad del sector.

\section{CONCLUSIONES Y RECOMENDACIONES}

El reconocimiento del valor del sector agrario obliga, desde años atrás, a la realización de un ejercicio de redireccionamiento estratégico, para generar actividades que dinamicen e incrementen incrementar los ámbitos económico y productivo, aumentando la competitividad en el agro colombiano. Para aumentar la competitividad en este renglón económico por medio de mayor calidad, mejores rendimientos y posición en los mercados, es necesario incorporar y apropiar nuevos insumos, técnicas y métodos, que incrementen la eficiencia y la productividad de las cadenas productivas y la comercialización del plátano en el Urabá antioqueño; es por ello por lo que, se hace necesario contar con nuevos sistemas de financiación que garanticen los recursos requeridos para el cultivo en esta región.

La búsqueda de alternativas financieras es un proceso que se ejecuta de manera continua para estimular el desarrollo de nuevas empresas y la consolidación y crecimiento de las que ya existen; no obstante, el sector productivo colombiano aún enfrenta un sinnúmero de barreras y dificultades para el acceso a los recursos financieros ofrecidos por el mercado; esto le ocurre principalmente a las pequeñas y medianas empresas. Del análisis del modelo presentado en este artículo se desprende la importancia de que hoy en día las empresas accedan cada vez más a las soluciones que los mercados financieros pueden ofrecerles [20]. El mecanismo de endeudarse mediante Titularización puede ser muy beneficioso para las estrategias de crecimiento de los pequeños y medianos productores de plátano en el Urabá antioqueño en el mediano plazo por lo que hay que fomentar que un número mayor de empresas y sectores utilicen dichos métodos. En este sentido, la titularización es un mecanismo competitivo frente a las demás alternativas de financiación existentes en el país, logrando llegar a traer más beneficios que los mecanismos tradicionales, dado que por medio de este encontramos menores gastos financieros, mayor liquidez, diminución de activos ociosos y es una herramienta accesible para todo tipo de compañías y personas. La Tasa Interna de Retorno (TIR) evidencia que la titularización es viable para financiar proyectos agrícolas, además de que puede llegar a ser atractivo para que los inversionistas adquieran títulos valores de este tipo en el momento de diversificar su portafolio de inversión.

Se recomienda dar a conocer y capacitar a los agricultores e inversionistas sobre este mecanismo de financiación, ya que no es muy utilizado por el desconocimiento que existe en el sector. La Titularización puede ser un mecanismo de financiación que traería beneficios a los productores de plátano que cuentan con escasez de recursos financieros y que no cuentan con la documentación requerida para acceder a los sistemas de crédito tradicional. Las garantías exigidas para los créditos formales en la actividad agropecuaria son más difíciles de cumplir, y en general los costos de transacción son más elevados en las áreas rurales. Por esta razón, la Titularización es un mecanismo óptimo para estos productores de plátano, garantizando sus flujos futuros por medio de sus cultivos de plátano.

\section{REFERENCIAS}

[1] J. Zuluaga, "El agro colombiano se consolidó como el motor de la economía nacional," Noticias Ministerio de Agricultura, 06-Jul-2018.

[2] H. J. Martínez Covaleda, C. F. Espinal, and Y. Peña Marín, La cadena del banano en Colombia: una mirada global de su estructura y dinamica 1991-2005. Ministerio de Agricultura y Desarrollo RuralMINAGRICULTURA, 2005.

[3] D. W. Turner and F. E. Rosales, Banana root system: towards a better understanding for its productive management. Bioversity International, 2005.

[4] E. Duque Grisales and J. A. Patiño Murillo, "El mercado de bonos de carbono y su aplicación para proyectos hidroeléctricos," Rev. CINTEX, vol. 18, pp. 131-143, 2013.

[5] E. Duport Jaramillo and S. Viveros Rubiano, "Titularización: Una nueva visión para el desarrollo del país," Trab. Grado Para Optar Al Tít. Abog. Pontif. Univ. Javer. Bogotá DC, 2001.

[6] J. Echavarría, "Condiciones Laborales y Productivas en Microempresas de Confección de la ciudad de Medellín,” Rev. CINTEX, vol. 20, no. 2, pp. 79-95, 2015.

[7] V. Mirović and D. Bolesnikov, "Application of asset securitization in financing agriculture in Serbia," Econ. Agric., vol. 60, no. 3, pp. 551-564, 2013.

[8] R. A. Arbeláez Pérez, E. A. Duque Grisales, and J. A. Patiño Murillo, "Relación de los principales mercados bursátiles durante y después de la crisis subprime con el mercado de renta variable colombiano," Mercatec, no. 55, pp. 14-24, 2018.

[9] H. C. Hurtado Macías, "Descripción de la agrocadena del plátano en Colombia," Tesis para optar al título de Administración de Empresas Agropecuarias, Universidad de La Salle, Bogotá D.C., 2016.

[10] IPC, "Urabá Antioqueño," Instituto Popular de Capacitación - Regiones. . 
[11] J. L. Cárdenas González, S. Zapata Henao, and J. Sánchez Torres, "Análisis productivo de plátano en alta densidad y su relación con la precipitación en Urabá," Rev. POLITÉCNICA, vol. 13, no. 24, pp. 2735, 2017.

[12] M. Migliorelli and P. Dessertine, "Time for new financing instruments? A market-oriented framework to finance environmentally friendly practices in EU agriculture," J. Sustain. Finance Invest., vol. 8, no. 1, pp. 1-25, 2018.

[13] L. J. Sanz, "LA TITULARIZACIÓN MÁS ALLÁ DE LA CRISIS.," Debates IESA, vol. 15, no. 4, 2010.

[14] C. Blanco Barón, “La Titularización En Colombia: Veinte Años De Regulación, 1993-2013," Rev. Econ. Inst., vol. 17 (32), pp. 259-294, 2015.

[15] C. A. Ospina, Á. D. Palomá, and D. F. Orjuela, "Proceso de titularización en cultivos de tardío rendimiento, palma de aceite.," Especialización, Colegio de Estudios Superiores en Administración, Bogotá D.C., 2008.

[16] C. Blanco Barón, "Titularizacion en Colombia: Diez Años de Desarrollo, La," Con-Texto, vol. 18, p. 7, 2004.

[17] Superintendencia Financiera de Colombia, Titularización - Patrimonio Autónomo - Vocero. 2007.

[18] E. Zapa Pérez, "Impacto de la Gestión por Procesos en la Innovación de las Organizaciones," Rev. CINTEX, vol. 19, pp. 23-37, 2014.

[19] T. Dwyer, T. A. Murphy, and B. Lim, Advancing the securitisation of Australian agriculture: Hybrid Equity. Rural Industries Research and Development Corporation, 2004.

[20] E. A. Duque-Grisales, J. M. Flórez, and N. O. Núñez, "Operación del sistema de autocontrol y gestión del riesgo de lavado de activos y financiación del terrorismo en empresas del sector comercial," Rev. CINTEX, vol. 23, no. 1, pp. 32-42, 2018. 